\title{
ROLE OF GUT MICROBIOME IN IMPROVING ANIMAL HEALTH AND PRODUCTIVITY
}

\author{
P. DAS ${ }^{1 *}$ AND R. RANJAN ${ }^{2}$ \\ ${ }^{1}$ Department of Veterinary Anatomy,West Bengal University of Animal and Fishery Sciences, \\ Kolkata-700 037, West Bengal, India \\ ${ }^{2}$ Department of Veterinary Anatomy, College of Veterinary Sciences and Animal Husbandry, \\ N.D.V.S.U, Rewa-486 001, Madhya Pradesh, India
}

\begin{abstract}
The gut microbiome comprises of a complex combination of microbes which includes archaea, bacteria, fungi, protozoa, helminths and viruses. The mammalian gastro-intestinal tract harbors this complex ecosystem in equilibrium with the host immune system. This organized and long-lasting process of colonization by microorganisms has developed into flourishing relationships creating diverse environment within the host gut. The most magnificent feature of this commensal relationship is that the host requires commensal microorganisms to get colonized in gastrointestial tract (GIT) for its own development and health. The present review article will focus on various aspects of gut microbial colonization and their influence on the host organ systems in relation to its role in various morphological changes occurring within the host leading to improvement in health and productivity of animals.
\end{abstract}

Key words: Gut microbiome, Gut-organ axis, Morphological changes, Productivity

\section{Introduction}

A diverse microbial population colonizes the in-utero sterile mammalian gastrointestinal tract during and after the birth. This organized and long-lasting process of colonization by microorganisms has developed into flourishing relationships creating diverse environment within the host gut (Mazmanian et al., 2005). There are various factors that affect the optimum colonization of gut microbiome and their normal functioning on various organ systems of the host body directly or indirectly. The gut microbiome comprises of a complex combination of microbes (Mezouar et al., 2018). The mammalian gastro-intestinal tract harbors this complex ecosystem in equilibrium with the host immune system. The most magnificent feature of this commensal relationship is that the host requires the commensal microorganism to get colonized in their gastrointestinal tract for their own development and health. The process of colonization is a two-way interaction between the host and the microorganisms.

\section{Colonization of gut microbiota in the host gastrointestinal tract}

During intra-uterine life, the gastrointestinal tract (GIT) of the developing fetus is almost sterile that gets colonized by microorganisms gradually during and after birth. This colonization is a two-way interaction between the host and the microbiome. The host factors such as intestinal $\mathrm{pH}$, retention of food particles in the gut and host immune system and the 
microbial factors like adhesion with the gut wall, survivality in the diverse environment within the gut and nutrient dependence as well as external factors such as diet and antibiotic treatment during early life, all combine to affect the gut colonization. Gut microbes of ruminants, specially the rumen microbiome, provide $70 \%$ of their daily energy requirement from the fermentation of indigestible dietary substrates. Therefore, studies in ruminant gut microbiota have focused mainly on the rumen to understand how this microbiome affects meat and milk production (Malmuthuge et al., 2015).

Rumen microbiota is involved in the fermentation of complex carbohydrates, and their composition is influenced by a number of factors. Rumen microbial composition varies significantly depending on the species, diet, host age, season, and geographic region. Bacteria dominate the ruminal microbial population and responsible for the production of volatile fatty acids (VFAs) and microbial proteins. Anaerobic bacteria dominate the rumen of neonatal ruminants by the second day of life $\left(10^{9} \mathrm{CFU} /\right.$ $\mathrm{mL}$ of rumen fluid) and the density of cellulolytic bacteria is stabilized $\left(10^{7} \mathrm{CFU} / \mathrm{mL}\right.$ of rumen fluid) within the first week of life. The establishment of other microbial groups is delayed until after bacterial establishment (Fonty et al., 1987).

Anaerobic fungi and methanogens appear in the neonatal rumen between 8 and 10 days postpartum (Fonty et al., 1987), while protozoa appear only after 15 days postpartum (Fonty et al., 1988). The early rumen microbiote consists of bacterial species from Proprionibacterium, Clostridium, Peptostreptococcus and Bifidobacterium, while Ruminococcus species dominates the cellulytic bacterial population (Fonty et al., 1987). When bacterial population throughout the gut was analyzed, the rumen and large intestinal regions consisted primarily of Bacteroidetes and Firmicutes, while $>95 \%$ of the bacteria in the small intestine contents were composed of Firmicutes. In contrast, the mucosa-associated bacterial community in the small intestine is composed of primarily Bacteroidetes, Firmicutes, and Proteobacteria, including 17 genera that are unique to this region of the intestine (Malmuthuge et al., 2014).

\section{Keynote factors affecting gut microbiome colonization}

In animals, the host and the microbiome can have a symbiotic as well as pathogenic association. Symbiotic association is beneficial to both the host and the microbes. The symbionts need not to completely depend on the host and on the other hand, the host can have different symbionts at different times. This host-microbe interaction is affected by a number of factors that involves the immune system of the host, nutrition, reproduction and many others (Bahrndorff et al., 2016). The climate and seasonal changes also influence the gut microbiome. A strong variation in the gut microbiota of chicken has been observed depending upon the season where the microbial population in summer was double than that of in winter (Carrasco et al., 2019). On the other hand, many studies reported differences in the gut microbiota profiles of poultry according to geographic location (Pandit et al., 2018). The farm managemental factors also play a key role in influencing the gut microbiome population.

\section{Gut microbiome and host digestive system}

The main function of the gastrointestinal tract microbes is digestion of ingested food substrates. The symbiotic relationship between the microbes and their host primarily involves acquisition of nutrients. However, beyond digestion, GIT microbes perform other functions that potentially contribute to the overall health status of the host (Hanning and Diaz-Sanchez, 2015). Hooper et al. (2001) reported that Bacteroides thetaiotaomicron 
modulates 71 intestinal genes involved in various processes including intestinal maturation and nutrient absorption. Experiments have suggested the role of gut microbiome in digestion and metabolism of nutrients and thus helps in weight management in human and animals. Lactobacillus spp. are found to be involved in the digestion of complex carbohydrates not digested by the host and also participate in the degradation of lipids and simple sugars. Moreover, Lactobacillus sp. are able to survive in the gut at low $\mathrm{pH}$ and bile and further produce certain antimicrobial agents with the help of which they reduce the number of bacteria in the gut, thus significantly affect the gut microbiome and consequently on weight change (Drissi et al., 2017).

Drastic differences are observed in germfree animals. Rekecki et al. (2009) reported reduction of mucosal linings due to absence of microbial stimulation of mucosa production in germfree sea bass (Centropristis striata). The intestinal villi of the germfree dog have been reported to be of same length as the normal dog but with thinner and pointer tips (Heneghan et al., 1979). The lamina propria of germfree mice had a sparse stroma, with few lymphocytes and macrophages, and the Peyer's patches were smaller. In all germfree animals studied as reported by authors, the turnover rate of intestinal epithelium was decreased. These changes in the histology of intestine are the results of a reduced interaction with the bacteria that stimulate the immune system and histological development (Fasano and Sheadonoghue, 2005). Apart from histological changes in the intestine, changes in other organs have also been reported. The germfree rat had a smaller heart while the cecum was four to six times larger than rats with normal microbiomes (Gordon et al., 1963). This change has been explained by an accumulation of substances normally degraded by the microbiome (Ishikawa et al., 1989). In germfree chicks, a change in the normal body temperature was noted which was slightly higher than that of chicks with normal microflora (Harrison and Hewitt, 1978).

These experiments suggest that the microbiome play a complex role in the host development. Age of the host also play a critical role in changing the microbial population. For example, the chicken gastrointestinal tract is sterile at hatch but gets quickly inhabited by aerobic Proteobacteria and after 12 days it gets dominated by anaerobic Firmicutes. Initially, the Proteobacteria stimulate the histological maturation of the gastrointestinal tract and thus provide an ideal environment for the Firmicutes to establish within the gut. The Proteobacteria grow well in the immature gastrointestinal tract but are poor competitors and are replaced after a mature and anaerobic gut environment is established. The successive colonization of bacteria also depends on nutrition and gut bacteria population selection. This establishment can also be selected by feeding specific foods. This was demonstrated in rabbits fed only milk for the first 42 days of life. These animals lacked the cellulolytic bacteria in the cecum and could not digest plant feeds (Padilha et al., 1999).

Modification of intestinal microbiome can promote growth in many agricultural animals. Delivery of probiotic cultures to animals can enhance the development of the intestinal tract (Callaway et al., 2008). The mature gastrointestinal tract improves nutrient uptake and increases the growth rate of the animal. Careful selection of probiotic bacteria is crucial as it can have a lifelong impact on intestinal health. Shirkey et al. (2006) reported differences in the gut histology and immune marker responses dependent on which bacteria initially colonized the gut of the pigs. The authors reported that the microbial diversity is important for healthy maturation of the entire intestinal tract. 


\section{Gut-microbiome-immunity axis in health}

The mammalian immune system is a dynamic and remarkable organ which recognizes, responds and adapts countless foreign and self molecules thus acts as a primary force to the process of health and disease (Janeway et al., 2001). Commensal bacteria have positive impact on development of gut-associated lymphoid tissues (GALT), intraepithelial lymphocytes and production of mucosal IgA (Hooper, 2004). Dendritic cells loaded with commensal bacteria that induce responses to commensals do not penetrate further than the draining mesenteric lymph nodes, immune induction to commensals is confined to mucosa, and thus the protective mucosal immunity to commensals is separated from systemic immunity (Macpherson et al. 2005). In this way the systemic immune system is kept completely ignorant of commensals.

The gastrointestinal tract represents a major gateway for infection by potential pathogens. Accordingly, the gut mucosa has evolved multiple layers of protection against invading pathogens. Epithelial cells have tight junctions and are covered with layers of mucus produced by goblet cells. The intestinal epithelium contains a large population of intra-epithelial lymphocytes (IELs), which recognize and eliminate infected or damaged epithelial cells. Paneth cells recognize microbial products via pattern recognition receptors, including Tolllike receptors (TLRs) and nucleotide-binding oligomerization domain (NOD)-like receptors and contribute to innate immunity by secreting a diverse repertoire of antimicrobial proteins. In the lamina propria, dendritic cells (DCs) extend processes between the epithelial cells to continuously monitor the content of the gut lumen and activate lymphocytes. The lamina propria contains large numbers of effector lymphocytes even in the absence of disease. These include immunoglobulin A ( $\operatorname{Ig} \mathrm{A}$ )- producing plasma cells and $\gamma \delta \mathrm{T}$ cells. In addition, recent studies have revealed that $\mathrm{CD}^{+} \mathrm{T}$ cells in the intestinal mucosa comprise significant numbers of Interleukin (IL)-17-expressing (Th17) cells. Furthermore, the mucosal immune system contains other unusual lymphocytes, such as IL-22-producing natural killer-like cells, which are positive for NK cell markers including NKp46 but do not have the killing activity of MHC class I negative cells and $\mathrm{CD}^{+}{ }^{+} \mathrm{CD} 3^{-}$ lymphoid tissue inducer cells (LTi cells), which are known to orchestrate the development of programmed and induced intestinal lymphoid tissues (Tanoue et al., 2010).

All these cells remain in sufficient amount in the intestinal mucosa even under normal conditions as there is always a chance of deleterious responses to commensal microbiota. Shortly after birth, the bacterial microflora forms an ecosystem in dynamic equilibrium. This beneficial relationship can be maintained only when the host immune system discriminates the commensal organism from the pathogenic one, and thereby exerts immune regulation by inducing inhibitory molecules for innate immune signaling, as well as inducing the accumulation of regulatory $\mathrm{T}$ (Treg) cells.

\section{Microbiota-gut-brain axis}

It has been reported by Li et al. (2018) that the microorganisms and circadian clock genes can interact with each other and can regulate insomnia, circadian rhythm disorders and depression, host sleep and mental states through the microbiome-gut-brain axis. Emotion and physiological stress can also affect the composition of the gut microorganisms. The gut microbiome and inflammation may be linked to sleep loss, circadian clock misalignment, affective disorders, and metabolic disease. The microbiome-gut-brain (MGB) axis (Bercik, 2011) affects brain function through three pathways that produce a bidirectional flow of 
information. The first of these is the immunoregulatory pathway, in which the microbiota interacts with immune cells in such a way as to affect the levels of cytokines, cytokinetic reaction factor, and prostaglandin E2 (Feng et al., 2018). As a result, brain function is affected. The second is the neuroendocrine pathway. Raybould (2010) reported that there are more than 20 types of enteroendocrine cells in the intestine, which constitutes one of the largest endocrine organs in the body. Powley et al. (2008) observed that the gut microbiota regulates the secretion of neurotransmitters such as cortisol, tryptophan, and serotonin (5-HT) and thereby affect the hypothalamic-pituitary-adrenal (HPA) axis and the central nervous system (CNS). The third is the vagus nerve pathway, in which the enteric nervous system plays an important role. The sensory neurons of the intestinal myenteric plexus are exposed to the gut microbiota; these neurons form synaptic contacts with motor neurons in the intestine and thus regulate the intestinal motility and gut hormone secretion. The intestinal nervous system also forms synapses with the vagus nerve, which connects the intestine to the brain (Powley et al., 2008) and constitutes an information transmission pathway that could be described as the gut microbiota-enteric nervous system (ENS)vagus-brain pathway. Wang and kasper (2014) reported that the gut microbiome produces neurotoxic metabolites such as D-lactic acid and ammonia that may pass through the vagus nerve into the CNS and may effect the brain function, stress responses, and sleep structure. Similarly, the CNS can also regulate the intestinal microbiota composition through these three pathways.

\section{Microbial interaction with the gut-lung Axis} Trompette et al. (2014) reported that dysbiosis in gut microbiota is associated with lung disorders and respiratory infections. Asthma in early life has been correlated with reduction in genus Bifido bacteria and increase in Clostridia in the intestine (Kalliomaki et al., 2001). Further studies have shown that the antibiotic intake depletes the population of certain species within the gut microbiota and thus influences lung diseases and allergic inflammation (Metsälä et al., 2015). Looft and Allen (2012) further reported that removal of sensitive gut bacteria after administration of neomycin in mouse leads to an increase in the susceptibility to influenza virus infection in lungs.

Vice-versa phenomenon has also been reported where changes in lung microbiota may also influence the composition of gut microbiota. Looft and Allen (2012) had reported that influenza virus infection in the respiratory tract in mice increases Enterobacteriaceae and reduces Lactobacilli and Lactococci in the intestinal microbiota. Similarly, Sze et al. (2014) had reported that upon administration of lipopolysaccharide (LPS) in mice, the lung microbiota changes that further affects the gut microbiota due to movement of bacteria from lung into the bloodstream. Harris et al. (2006) stated that IgA positive plasma cells are in lesser numbers in neonates and germ-free animals. The immunity and IgA production enhances after colonization by gut bacteria. The $\mathrm{T}$ and $\mathrm{B}$ cells, induced in Peyer's patches, can move into the circulation and migrate to intestinal epithelium, bronchial epithelium and lymphoid tissues (Matsuno et al., 2010). Such B-cells produce $\operatorname{Ig}$ A which is transported to the mucosal surface and passes immunological information between different organs (Bingula et al., 2017). Thus, the lymph and/or bloodstream acts as a link between the gut (where primary sensitization occurs) and the affected site on the lung (He et al., 2017)

\section{The role of gut microbiome as a virtual endocrine organ}

The gut microbiome affects the host physiology. Manipulation of its composition influences the 
host metabolism and body composition. The virtual endocrine organs also play a role in the regulation of the plasma concentrations of tryptophan, a key neurotransmitter and precursor to serotonin, within both the enteric and central nervous systems. The gut microbiota also controls the hypothalamic-pituitary-adrenal axis. This is evident from studies in microbiotadeficient germ-free animals which showed exaggerated responses to psychological stress that was further normalized by monocolonization with certain bacterial species including Bifidobacterium infantis. Therapeutic targeting of the gut microbiota may thus be useful in treating or preventing stress-related microbiome-gut-brain axis disorders and metabolic diseases (O'Collaghan et al., 2016).

It has been reported that the gut microbiota plays a critical role in the production of norepinephrine and dopamine in the gut (Asano et al., 2012). This production is due to the expression of $\beta$-glucuronidases by commensal gut bacteria, generating dopamine and norepinephrine through the cleavage of their inactive conjugated forms. Gut microbes can also produce non-noradrenergic, noncholinergic transmitters such as nitric oxide, which plays a pivotal role in the regulation of gastric emptying (Orihata and Sarna, 1994), through the anaerobic reduction of nitrate to nitrogen (Cutruzzola, 2012). The inhibitory transmitter gamma-aminobutyric acid can be generated by Lactobacillus brevis and Bifidobacterium dentium (Barrett et al., 2012); both of these organisms were isolated from humans (Ventura et al., 2009). Researchers have shown that the gut microbiota plays a role in the development of diabetes (Wen et al., 2008). The gut microbiota, through LPS, has been shown to exert a pro-inflammatory effect and when combined with a high-fat diet it leads to the onset of insulin resistance and, therefore, the development of type 2 diabetes had been observed in a mouse model (Cani et al., 2008).
Studies have suggested the role of gut microorganism in modulating the levels of the serotonin precursor tryptophan and hence the microbiota has a control over serotonin levels in the host. Studies have shown that germ-free rats have depleted levels of tryptophan, but upon administration of certain bacteria, such as Bifidobacterium species, tryptophan levels are increased (Desbonnet et al., 2008), thus suggests that bacteria can alter the available serotonin pool and ultimately elicit communication between the gut and the brain. Some microbes, such as Candida spp., Streptococcus spp., Escherichia spp. and Enterococcus spp., have been shown to directly produce serotonin (Cryan and Dinan, 2012).

\section{Role of gut microbiome in animal productivity}

The gut microbial colony contains cellulolytic, hemicellulolytic, amylolytic, proteolytic, and lipolytic species, exhibiting a high level of functional redundancy, and is capable of effectively degrading host-indigestible plant fiber. Volatile fatty acids (VFAs), principally acetate, propionate, and butyrate, are the major products of rumen microbial fermentation and are absorbed and used as energy sources by the host. Ruminally derived VFAs can meet up to $70 \%$ of the host's energy needs, and thus their production is essential to animal performance. Metabolism of nitrogen-containing compounds (including peptides, ammonia and urea) by the rumen microbiota is also vital in the provision of microbial proteins to the host for muscle and milk synthesis. Ingested fiber, carbohydrates and protein are first hydrolyzed to shorter chains (oligomers) and monomers (glucose, amino acids) by primary members of the microbiota and subsequently used as substrates by various members of the microbial community (Hara et al., 2020).

Livestock industries are a significant source of environmentally harmful green house gases 
(GHG), with $\mathrm{CO}_{2}, \mathrm{CH}_{4}$, and $\mathrm{N}_{2} \mathrm{O}$ being the major green-house gases emitted from food and agricultural production chains. The potent global warming potential of $\mathrm{CH}_{4}$ is the most extensively studied GHG in terms of ruminant emissions, and reducing rates of enteric methanogenesis is desirable in terms of both improved animal productivity and environmental stability. The $\mathrm{CH}_{4}$ is produced in the rumen by methanogenic archaea, which are estimated to account for $0.3-3.3 \%$ of the rumen microbial population, based on $16 \mathrm{~S}$ ribosomal RNA (rRNA) gene analysis (Janssen and Kirs, 2008). Three major pathways of ruminal methanogenesis have been reported (Hungate, 1967; Leahy et al., 2013) out of which, hydrogenotrophic methanogenesis is the predominant pathway in the rumen and is carried out mainly by Methanobrevibacter species. In relation to this, the most effective mitigation strategy demonstrated to date is basal dietary supplementation with 3-nitroproxypropanol (3-NOP). Developed in 2012, 3-NOP acts by inhibiting the methyl coenzyme-M reductase (MCR) enzyme in the terminal step of methanogenesis (Duval and Kindermann, 2012). Supplementation of 3-NOP has been shown to dramatically reduce ruminal $\mathrm{CH}_{4}$ production in lactating dairy cows and crucially does not have any adverse effect on milk yield (Hristov et al., 2015), though an increase in milk fat has been reported (Lopes et al., 2016). It has also been proven to be an effective $\mathrm{CH}_{4}$ inhibitor in sheep (Martínez-Fernández et al., 2014) and beef cattle (Romero-Perez et al., 2015).

\section{Conclusion}

Extensive studies have been reported on the

\section{REFERENCES}

Asano Y, Hiramoto T, Nishino R, Aiba Y, Kimura T et al., 2012. Critical role of gut microbiota in the production of biologically active, free catecholamines in the gut lumen of mice. Am J Physiol Gastrointest Liver Physiol, 303(11): G1288-G1295, doi: 10.1152/ajpgi.00341.2012 association between the host and gut in humans but the information regarding host-microbe relationship in animals is still in infant stage. However, the studies reviewed till date is generating fruitful results. The gut microbial composition and its influence on animal health and productivity needs a vast indepth identification of this complex ecosystem which will provide an insight into the diagnosis and treatment of autoimmune and auto inflammatory diseases in animals. The study of this complex interaction between the host and gut microbiota will further help to design new therapies for diseases arising due to physical and psychological stress. A substantial step forward will be helpful to explain the role of the gut microbiome in host physiology and to understand the metabolic capacity of the early microbiome. Isolation of metabolically active rumen microbiota may be important to enhance our understanding of their roles in monocultures and mixed populations. This information will provide the basis for future strategies to manipulate the microbiome and improve both production and health. The study of this interaction can further help the researchers to develop animal models that can provide information regarding microbial changes within specific regions of gastrointestinal tract and analyze local effects on mucosal immune functions. It can also be helpful to establish the information of effect of dysbiosis of microbiome during early colonization in newborn gut both locally and systemically as well as whether the restoration of gut flora during later stage can reverse the effect of early microbial dysbiosis that impacts the animal health and production.

Bahrndorff S, Alemu T, Alemneh T and Nielsen JL, 2016. The microbiome of animals: implications for conservation biology. Int J Genomics, 2016: 5304028, doi: 10.1155/2016/5304028

Barrett E, Ross RP, O’Toole PW, Fitzgerald GF and 
Stanton C, 2012. $\gamma$-aminobutyric acid production by culturable bacteria from the human intestine. J Appl Microbiol, 113(2): 411417, doi: 10.1111/j.1365-2672.2012.05344.x

Bercik P, 2011. The microbiota-gut-brain axis: learning from intestinal bacteria. Gut, 60(3): 288290, doi: 10.1136/gut.2010.226779

Bingula R, Filaire M, Radosevic-Robin N, Bey M, Berthon JY et al., 2017. Desired turbulence? Gutlung axis, immunity, and lung cancer. J Oncol, 50: 353-371, doi: 10.1155/2017/5035371

Callaway TR, Edrington TS, Anderson RC, Harvey RB, Genovese KJ et al., 2008. Probiotics, prebiotics and competitive exclusion for prophylaxis against bacterial disease. Anim Health Res Rev, 9(2): 217-225, doi: 10.1017/ S1466252308001540

Cani PD, Bibiloni R, Knauf C, Waget A, Neyrinck AM et al., 2008. Changes in gut microbiota control metabolic endotoxemia-induced inflammation in high-fat diet-induced obesity and diabetes in mice. Diabetes, 57(6): 1470-1481, doi: 10.2337/ db07-1403

Carrasco JMD, Natalia AC and Mariano EFM, 2019. Microbiota, gut health and chicken productivity: what is the connection. Mcroorganisms, 7(10): 374-388, doi: 10.3390/ microorganisms7100374

Cryan JF and Dinan TG, 2012. Mind-altering microorganisms: the impact of the gut microbiota on brain and behaviour. Nat Rev Neurosci, 13(10): 701-712, doi: 10.1038/nrn3346

Cutruzzola F, 2012. Nitric oxide synthesis by bacterial cytochrome $\mathrm{cd}_{1}$ nitrite reductases. Nitric Oxide, 27 (Suppl): S10, doi: 10.1016/j.niox.2012.04.037

Desbonnet L, Garrett L, Clarke G, Bienenstock J and Dinan TG, 2008. The probiotic Bifidobacterium infantis: an assessment of potential antidepressant properties in the rat. J Psychiatr Res, 43(2): 164-174, doi: 10.1016/ j.jpsychires.2008.03.009

Drissi F, Raoult D and Merhej V, 2017. Metabolic role of Lactobacilli in weight modification in humans and animals. Microb Pathog, 106: 182-194, doi: 10.1016/j.micpath. 2016.03.006
Duval S and Kindermann M, 2012. Use of nitrooxy organic molecules in feed for reducing enteric methane emissions in ruminants, and/or to improve ruminant performance. Patent No.WO2012085629A1

Fasano A and Shea-donoghue T, 2005. Mechanisms of disease: the role of intestinal barrier function in the pathogenesis of gastrointestinal autoimmune diseases. Nat Clin Pract Gastroenterol Hepatol, 2(9): 416-422, doi: 10.1038/ncpgasthep0259

Feng Q, Chen WD and Wang YD, 2018. Gut microbiota: an integral moderator in health and disease. Front Microbiol, 9: 151, doi: 10.3389/fmicb.2018.00151

Fonty G, Gouet P, Jounay JP and Senaud J, 1987. Establishment of the microflora and anaerobic fungi in the rumen of lambs. J Gen Microbiol, 133: 1835-1843, doi: 10.1099/00221287-1337-1835

Fonty G, Senaud J, Jouany JP and Gouet P, 1988. Establishment of ciliate protozoa in the rumen of conventional and conventionalized lambs: influence of diet and management conditions. Can J Microbiol, 34(3): 235-241, doi: 10.1139/ m88-044

Gordon HA, Wostmann BS and Bruckner- Kardoss E, 1963. Effects of microbial flora on cardiac outputs and other elements of blood circulation. Proc Soc Exp Biol Med, 114: 301-304, doi: 10.3181/ 00379727-114-28658

Hanning I and Diaz-Sanchez S, 2015. The functionality of the gastrointestinal microbiome in nonhuman animals. Microbiome, 3(1): 51-61, doi: 10.1186/s40168-015-0113-6

Hara E, Neves ALA, Song Y and Guan LL, 2020. The role of the gut microbiome in cattle production and health: driver or passenger? Annu Rev Anim Biosci, 8: 199-220, doi: 10.1146/annurevanimal-021419-083952

Harris NL, Spoerri I, Schopfer JF, Nembrini C, Merky $\mathrm{P}$ et al., 2006. Mechanisms of neonatal mucosal antibody protection. J Immunol, 177(9): 62566262, doi: 10.4049/jimmunol.177.9.6256

Harrison GF and Hewitt D, 1978. The influence of the conventional microbiota on the body temperature of the chicken. Br Poult Sci, 19(3): 
273-275, doi: 10.1080/00071667808416475

He Y, Wen Q, Yao F, Xu D, Huang Y et al., 2017. Gutlung axis: the microbial contributions and clinical implications. Crit Rev Microbiol, 43(1): 81-95, doi: 10.1080/1040841X.2016.1176988

Heneghan JB, 1979. Enterocyte kinetics mucosal surface area and mucus in gnotobiotes. In: Fliedner T, Heit H, Niethammer D, Pflieger H, editors. Clin Exp Genobiot. New York: Gustav Fishe, pp19-27

Hooper LV, Wong MH, Thelin A, Hansson L, Falk PG et al., 2001. Molecular analysis of commensal host-microbial relationships in the intestine. Science, 291(5505): 881-884, doi: 10.1126/ science.291.5505.881

Hooper LV, 2004. Bacterial contributions to mammalian gut development. Trends Microbiol, 12(3): 129-134, doi: 10.1016/j.tim.2004.01.001

Hristov AN, Oh J, Giallongo F, Frederick TW and Harper MT, 2015. An inhibitor persistently decreased enteric methane emission from dairy cows with no negative effect on milk production. Proc Natl Acad Sci, 112 (34): 10663-10668, doi: 10.1073/ pnas. 1504124112

Hungate RE, 1967. Hydrogen as an intermediate in the rumen fermentation. Arch Mikroboil, 59 (1): 158-164, doi: 10.1007/BF00406327

Ishikawa K, Satoh Y, Oomori Y, Yamano M, Matsuda M et al., 1989. Influence of conventionalization on cecal wall structure of germ-free Wistar rats: quantitative light and qualitative electron microscopic observations. Anat Embryol, 180: $191-198$

Janeway CA, Travers $P$, Walport $M$ and Shlomchik M, 2001. Immunobiology (New York: Garland Publishing). Chapter-3. https:// www.ncbi.nlm.nih.gov/books/NBK10757/

Janssen PH and Kirs M, 2008. Structure of the archaeal community of the rumen. Appl Environ Microbiol, 74(12): 3619-3625, doi: 10.1128/ AEM.02812-07

Kalliomaki M, Kirjavainen P, Eerola E, Kero P, Salminen S et al., 2001. Distinct patterns of neonatal gut microflora in infants in whom atopy was and was not developing. J Allergy Clin
Immunol, 107(1): 129-134, doi: 10.1067/ mai.2001.111237

Leahy SC, Kelly WJ, Ronimus RS, Wedlock N, Altermann E et al., 2013. Genome sequencing of rumen bacteria and archaea and its application to methane mitigation strategies. Animal, 7(Suppl.2): 235-243, doi: $10.1017 /$ S1751731113000700

Li Y, Hao Y, Fan F and Zhang B, 2018. The role of microbiome in insomnia, circadian disturbance and depression. Front Psychiatry, 9: 669, doi: 10.3389/fpsyt.2018.00669

Looft T and Allen HK, 2012. Collateral effects of antibiotics on mammalian gut microbiomes. Gut Microbes, 3(5): 463-467, doi: 10.4161/ gmic. 21288

Lopes JC, de Matos LF, Harper MT, Giallongo F and Oh J, 2016. Effect of 3-nitrooxypropanol on methane and hydrogen emissions, methane isotopic signature, and ruminal fermentation in dairy cows. J Dairy Sci, 99 (7): 5335-5344, doi: $10.3168 /$ jds.2015-10832

Macpherson AJ, Geuking MB and McCoy KD, 2005. Immune responses that adapt the intestinal mucosa to commensal intestinal bacteria. Immunol, 115(2): 153-162, doi: 10.1111/j.13652567.2005.02159.x

Malmuthuge N, Griebel PJ and Guan LL, 2015. The gut microbiome and its potential role in the development and function of newborn calf gastrointestinal tract. Front Vet Sci, 2: 36, doi: 10.3389/fvets.2015.00036

Malmuthuge N, Griebel PJ and Guan LL, 2014. Taxonomic identification of commensal bacteria associated with the mucosa and digesta throughout the gastrointestinal tracts of preweaned calves. Appl Environ Microbiol, 80(6): 2012-2028, doi: 10.1128/AEM.03864-13

Martínez-Fernández G, Abecia L, Arco A, Cantalapiedra-Hijar G and Martin-García AI, 2014. Effects of ethyl-3-nitrooxy propionate and 3-nitrooxypropanol on ruminal fermentation, microbial abundance, and methane emissions in sheep. J Dairy Sci, 97(6): 3790-3799, doi: 10.3168/jds.2013-7398

Matsuno K, Ueta H, Shu Z, Xue-Dong X, Sawanobori Y et al., 2010. The microstructure of secondary 
lymphoid organs that support immune cell trafficking. Arch Histol Cytol, 73(1): 1-21, doi: 10.1679/aohc.73.1

Mazmanian SK, Liu CH, Tzianabos AO and Kasper DL, 2005. An immunomodulatory molecule of symbiotic bacteria directs maturation of the host immune system. Cell, 122(1): 107-118, doi: 10.1016/j.cell.2005.05.007

Metsälä J, Lundqvist A, Virta LJ, Kaila M, Gissler M et al., 2015. Prenatal and post-natal exposure to antibiotics and risk of asthma in childhood. Clin Exp Allergy, 45(1): 137-145, doi: 10.1111/ cea. 12356

Mezouar S, Chantran Y, Micheld J, Fabre A, Dubusa JC et al., 2018. Microbiome and the immune system: From a healthy steady-state to allergy associated disruption. Human Microb J, 10: 11-20, doi: 10.1016/j.humic.2018.10.001

O'Callaghan TF, Ross RP, Stanton C and Clarke G, 2016. The gut microbiome as a virtual endocrine organ with implications for farm and domestic animal endocrinology. Domest Anim Endocrinol, 56(S): S44-S55, doi: 10.1016/ j.domaniend.2016.05.003

Orihata M and Sarna SK, 1994. Inhibition of nitric oxide synthase delays gastric emptying of solid meals. J Pharmacol Exp Ther, 271(2): 660-670

Padilha MTS, Licois D, Gidenne T and Carré B, 1999. Caecal microbiota and fermentation pattern in exclusively milk-fed young rabbits. Reprod Nutr Dev, 39: 223-230

Pandit RJ, Hinsu AT, Patel NV, Koringa PG, Jakhesara SJ et al., 2018. Microbial diversity and community composition of caecal microbiota in commercial and indigenous Indian chickens determined using 16S rDNA amplicon sequencing. Microbiome, 6(1): 115, doi: 10.1186/s40168-018-0501-9

Powley TL, Wang XY, Fox EA, Phillips RJ, Liu LW et al., 2008. Ultrastructural evidence for communication between intramuscular vagal mechanoreceptors and interstitial cells of Cajal in the rat fundus. Neuro gastroenterol Motil, 20(1): 69-79, doi: 10.1111/j.1365-2982.2007.00990.x

Raybould HE, 2010. Gut chemosensing: interactions between gut endocrine cells and visceral afferents.
Auton Neurosci, 153(1-2): 41-46, doi: 10.1016/ j.autneu.2009.07.007

Rekecki A, Dierckens K, Laureau S, Boon N, Bossier P et al., 2009. Effects of germ-free rearing environment on larval sea bass (Dicentrarchus labrax L.). Aquaculture, 293(1): 8-15, doi: 10.1016/ j.aquaculture.2009.04.001

Romero-Perez A, Okine EK, McGinn SM, Guan LL and Oba M, 2015. Sustained reduction in methane production from long-term addition of 3nitrooxypropanol to a beef cattle diet. J Anim Sci, 93(4): 1780-1791, doi: 10.2527/jas.2014-8726

Shirkey TW, Siggers RH, Goldade BG, Marshall JK, Drew MD et al., 2006. Effects of commensal bacteria on intestinal morphology and expression of proinflammatory cytokines in the gnotobiotic pig. Exp Biol Med, 231(8): 1333-1345, doi: 10.1177/ 153537020623100807

Sze MA, Tsuruta M, Yang SW, Oh Y, Man S et al., 2014. Changes in the bacterial microbiota in gut, blood, and lungs following acute LPS instillation into mice lungs. PLoS One, 9(10): e111228, doi: 10.1371/journal.pone. 0111228

Tanoue T, Umesaki Y and Honda K, 2010. Immune responses to gut microbiota-commensals and pathogens. Gut Microbes, 1(4): 224-233, doi: 10.4161/gmic.1.4.12613

Trompette A, Gollwitzer ES, Yadava K, Sichelstiel AK, Sprenger N et al., 2014. Gut microbiota metabolism of dietary fiber influences allergic airway disease and hematopoiesis. Nat Med, 20(2): 159-166, doi: 10.1038/nm.3444

Ventura M, Turroni F, Zomer A, Foroni E, Giubellini V et al., 2009. The Bifidobacterium dentium Bd1 genome sequence reflects its genetic adaptation to the human oral cavity. PLoS Genet, 5(12): 785, doi: 10.1371/journal.pgen.1000785

Wang Y and Kasper LH, 2014. The role of microbiome in central nervous system disorders. Brain Behav Immun, 38: 1-12, doi: 10.1016/j.bbi.2013.12.015

Wen L, Ley RE, Volchkov PY, Stranges PB, Avanesyan L et al., 2008. Innate immunity and intestinal microbiota in the development of type 1 diabetes. Nature, 455(7216): 1109-1113, doi: 10.1038/ nature 07336

Received-15.09.2020, Accepted - 27.10.2020, Published - 01.12.2020 